\title{
Treatment of Refractory Ascites
}

\author{
Harjit Bhogal, M.D. and Arun J. Sanyal, M.D.
}

Ascites is the accumulation of abnormal amounts of fluid in the peritoneal cavity. Cirrhosis is a frequent cause of ascites, but ascites can also result from malignant or infectious processes that involve the peritoneal cavity. The onset of ascites in patients with cirrhosis is associated with substantial increases in morbidity and mortality. Ascites not only is associated with physical discomfort but also can cause shortness of breath, umbilical hernias, and asthenia. It can be complicated by hepatic hydrothorax, spontaneous bacterial peritonitis, hyponatremia, and renal dysfunction. Ascites due to cirrhosis is primarily managed with salt restrictions, diuretics, and intermittent paracentesis as needed for symptom relief. When ascites cannot be mobilized with sodium restrictions and diuretics, it is considered to be refractory to medical treatment.

\section{How Is Refractory Ascites Defined? ${ }^{1}$}

There are two operational definitions of refractory ascites: (1) ascites that recurs after paracentesis or cannot be mobilized despite maximal medical treatment and (2) ascites that recurs or cannot be mobilized because of an inability to use effective doses of diuretics due to the development of diuretic-associated adverse events. Maximal medical treatment is generally defined as a sodium restriction of less than $2 \mathrm{~g} /$ day, the use of a maximal dose of a loop-acting diuretic (160 mg of furosemide per day or the equivalent dose of another loop-acting diuretic), and the use of a distal tubule-acting diuretic (400 mg of spironolactone per day or an equivalent dose of another distal tubule-acting diuretic). It is important to ensure compliance with sodium restrictions before ascites is considered to be refractory to medical treatment. If the 24-hour urine sodium level is $>78 \mathrm{mEq}$ and the patient is not losing weight, noncompliance with sodium restrictions should be addressed. A failure to control ascites due to excessive sodium intake does not constitute refractory ascites.

\section{Approaches to Difficult-To-Manage Ascites and Diagnosis of Refractory Ascites}

\section{Differential Diagnosis}

There are several possibilities that one must consider when ascites recurs rapidly after large-volume paracentesis or cannot be easily mobilized. Noncompliance with diuretics and sodium restrictions is a common cause of failures to remove ascites or its rapid recurrence after therapeutic paracentesis. This requires a careful history, and the urinary sodium output can be checked to confirm it. This requires the quantification of both the urine (sodium) and the volume. If the urinary sodium output is greater than that prescribed in the diet, the patient should have a negative sodium balance and will not be expected to retain sodium and water (i.e., ascites). When ascites accumulates in the face of a sodium output that is greater than what is supposedly being taken orally, this indicates noncompliance. The management of noncompliance involves educating the patient and his or her caregivers about the importance of compliance with sodium restrictions.

Another possibility is the development of hepatocellular cancer with either vascular involvement or spreading to the peritoneal surface. The development of a superinfection of the peritoneal cavity (e.g., tuberculous peritonitis) in areas in which this is prevalent may also make ascites difficult to mobilize. These possibilities can be easily excluded by the repetition of diagnostic paracentesis and the performance of right upper quadrant ultrasound with a Doppler examination of hepatic vessels. This step should be performed

Abbreviations: MELD, Model for End-Stage Liver Disease; TIPS, transjugular intrahepatic portosystemic shunt.

From the Division of Gastroenterology, Hepatology, and Nutrition, Department of Internal Medicine, Virginia Commonwealth University School of Medicine, Richmond, VA.

Potential conflict of interest: Nothing to report.

View this article online at wileyonlinelibrary.com

(c) 2013 by the American Association for the Study of Liver Diseases

doi: $10.1002 /$ cld.211 
relatively early in the evaluation of difficult-to-manage ascites. The development of hemorrhagic ascites, chylous ascites, or changes in the protein, lactate dehydrogenase, and cell counts of ascites should alert the health care provider to these possibilities.

Finally, the presence of heart failure or underlying chronic kidney disease may not allow adequate diuresis and may contribute to the refractoriness of ascites to medical therapy. The presence of these conditions is often clinically obvious, but sometimes additional testing is needed. Basal crackles, an accentuated pulmonary component of the second heart sound, tricuspid regurgitation, and jugular venous distension suggest underlying heart disease, whereas hematuria and proteinuria suggest the presence of underlying intrinsic kidney disease.

\section{Diagnostic Approach}

The early diagnostic assessment, therefore, includes a careful history and examination, a urinalysis including urine (sodium), a Doppler ultrasound examination, and a diagnostic paracentesis. An echocardiogram may be performed selectively on the basis of the clinical assessment. If alternative causes of recurrent or difficult-to-mobilize ascites are found, they should be treated accordingly. The doses of diuretics can be increased until the maximal doses are being given. It is common practice to increase the dose of spironolactone by $100 \mathrm{mg} /$ day and the dose of furosemide by $40 \mathrm{mg} /$ day. The doses of spironolactone and furosemide can be increased, with the dosing ratio of spironolactone to furosemide kept at 5:2 every 3 to 5 days and with close monitoring of daily weights and electrolytes. Whenever possible, both diuretics should be given together because of their synergistic effects on natriuresis in cirrhosis-related ascites. Spironolactone has a long half-life and should be given as a single daily dose, whereas furosemide is often given twice a day, particularly at higher doses. Large-volume paracentesis can be performed for symptom relief, and all ascites can be safely drained as long as albumin is administered intravenously at a dose of 6 to $8 \mathrm{~g} / \mathrm{L}$ of ascites removed. It is not imperative to administer albumin before the paracentesis, and it can be given concomitantly or shortly thereafter. When ascites recurs rapidly after therapeutic taps or cannot be mobilized despite maximal diuretic therapy or when effective diuretic dosing is not possible because of diuretic-related adverse events, a diagnosis of refractory ascites can be made.

\section{Treatment Options ${ }^{2}$}

Repeated Large-Volume Paracentesis. This is a widely available and easy initial approach that rapidly makes the patient comfortable. It does not, however, improve the basic pathophysiology of the disease process, and ascites frequently recurs.
Repeated paracentesis requires repeated visits to a health care facility and is associated with worsened quality of life. It also has no impact on survival, which is driven by the Model for EndStage Liver Disease (MELD) score in such patients.

Transjugular Intrahepatic Portosystemic Shunt $\left(\right.$ TIPS $^{3}$. Ascites is caused by cirrhosis-induced sinusoidal portal hypertension. TIPS is a procedure that decompresses the hypertensive portal vein and hepatic sinusoids without the need for major surgery. After TIPS, there is an increase in the venous return to the heart, which results in an increased output to perfuse the kidneys and a consequent decrease in proximal tubular sodium reabsorption; natriuresis is thereby produced. Maximal natriuresis may take several weeks after TIPS, and sodium restrictions and diuretics should be continued initially after TIPS. Diuretics may be decreased or even withdrawn on a select case-by-case basis according to the clinical response after TIPS when it is placed for refractory ascites.

Several trials have compared TIPS to medical treatment (sodium restriction, diuretics, and taps) for refractory ascites (see reference 3 for a more detailed review). TIPS is clearly superior to medical treatment for maintaining an ascites-free state. It is, however, controversial whether TIPS improves survival. The two largest and most rigorously performed trials failed to demonstrate a survival advantage for TIPS. TIPS is also associated with increased encephalopathy. In the trials that documented it, TIPS did not clearly reduce infections or the development of renal failure. It has been suggested that the risk of hepatocellular cancer increases after TIPS, but this remains to be validated.

The ideal patient for TIPS is one who has refractory ascites but has relatively preserved hepatic synthetic function and no or well-controlled encephalopathy. A MELD score greater than 18 is a risk factor for poor outcomes after TIPS, and patients with a MELD score above this value may not be served well by TIPS. In such cases, the decision to perform TIPS should be made on a case-by-case basis in conjunction with hepatologists with considerable experience with this procedure. Those with portopulmonary hypertension and known heart failure should not receive a TIPS because of the likelihood of worsened heart failure due to an increased preload after the procedure.

Liver Transplantation. All subjects with refractory ascites and a MELD score greater than 14 should be evaluated for liver transplantation and listed unless there is a contraindication for transplantation.

Aquaretic Drugs ${ }^{4}$. Refractory ascites is often complicated by the development of hyponatremia. This is due to severe water retention that exceeds the degree of sodium retention. 
Hyponatremia is managed initially with water restrictions. In severe and especially symptomatic cases (sodium level $<120$ $\mathrm{mEq} / \mathrm{L}$ ), one may consider the use of vasopressin antagonists such as tolvaptan and conivaptan. These increase free water excretion and rapidly improve hyponatremia. They are diuretics and may help to mobilize ascites. Their use is limited by cost and the potential for rapid increases in serum sodium, with damaging consequences on the central nervous system. Their routine use for the treatment of refractory ascites is considered experimental.

Peritoneovenous Shunts ${ }^{5}$. This procedure involves returning ascites to the central circulation by the placement of a catheter connecting the two compartments. It also increases venous return and effective perfusion of the kidneys. It is, however, frequently complicated by occlusion and the occasional development of severe coagulopathy. It can also precipitate heart failure in patients with subclinical or overt myocardial dysfunction. The shunts can also become infected and need to be removed. Finally, these shunts do not improve survival. These considerations have decreased enthusiasm for this procedure, which should be considered mainly as a palliative measure when TIPS is contraindicated and liver transplantation is not an option.

\section{References}

1. Salerno F, Guevara M, Bernardi M, Moreau R, Wong F, Angeli P, et al. Refractory ascites: pathogenesis, definition and therapy of a severe complication in patients with cirrhosis. Liver Int 2010;30:937-947.

2. Runyon BA. Management of adult patients with ascites due to cirrhosis: an update. Hepatology 2009;49:2087-2107.

3. Bhogal HK, Sanyal AJ. Using transjugular intrahepatic portosystemic shunts for complications of cirrhosis. Clin Gastroenterol Hepatol 2011;9:936-946.
Ancillary Measures. Patients with low-protein ascites, hyponatremia, a Child-Turcotte-Pugh score greater than 9, and/or an elevated serum creatinine are at increased risk of infection including the development of sponataneous bacterial peritonitis. Careful attention to nutritional status and infection prevention with the use of quinolones are essential ancillary measures in the management of refractory ascites in these high risk patients. ${ }^{6}$

What Not to Do. The placement of an indwelling peritoneal drainage catheter is strongly discouraged because of the risk of infection, which is likely to result in renal failure and also render a patient ineligible for liver transplantation.

In summary, the assessment and management of refractory ascites require a careful consideration of the potential mechanisms underlying the difficulty in mobilizing the ascites and a treatment based on the pathophysiology of the ascites. Liver transplantation remains the best long-term treatment for refractory ascites.

CORRESPONDENCE:

A. J. Sanyal, M.D., Division of Gastroenterology, Hepatology, and Nutrition, Department of Internal Medicine, Virginia Commonwealth University School of Medicine, MCV Box 980342, Richmond, VA 23298-0342. E-mail: asanyal@mcvh-vcu.edu

4. Ali F, Guglin M, Vaitkevicius P, Ghali JK. Therapeutic potential of vasopressin receptor antagonists. Drugs 2007;67:847-858.

5. Scholz DG, Nagorney DM, Lindor KD. Poor outcome from peritoneovenous shunts for refractory ascites. Am J Gastroenterol 1989;84: 540-543.

6. Gines P, Angeli P, Lenz K. EASL clinical practice guidelines on the management of ascites, spontaneous bacterial peritonitis, and hepatorenal syndrome in cirrhosis. J Hepatol 2010;53:397-417. 